\title{
A model reference approach for implementing active structural acoustic control
}

\author{
Robert L. Clark and Chris R. Fuller \\ Mechanical Engineering Department, Virginia Polytechnic Institute and State University, Blacksburg, \\ Virginia 24061
}

(Received 14 August 1991; revised 2 March 1992; accepted 29 April 1992)

\begin{abstract}
A model reference active structural acoustic control (ASAC) approach is investigated as a method for replacing microphone error sensors located in the acoustic field with accelerometers located on the structure. The suggested controller is the multi-input/multioutput adaptive LMS algorithm. As opposed to driving the response of the sensor located on the structure to zero with the control inputs, the response is driven to some pre-determined "reference" value corresponding to an a priori training cost function, which is originally chosen to minimize either the far-field pressure at a number of acoustic field points, or the supersonic region of the wave-number transform. In effect, the uncontrolled structure is adaptively modified to behave like the reference structure. Results indicate that the identical optimal control solution can be obtained when implementing the structural sensors, and the required number of sensors must simply equal the number of control actuators. In addition, for the example studied here, significant reduction in far-field sound radiation can be achieved even when errors of $\pm 5 \%$ are present in the reference model. A considerable level of attenuation in sound can also be achieved for a band of frequencies within $\pm 5 \%$ of the reference frequency used to obtain the models for creating the disturbance. Thus it appears that the model reference approach is reasonably robust.
\end{abstract}

PACS numbers: 43.40.Vn, 43.50.Ki

\section{INTRODUCTION}

One of the primary concerns in active structural acoustic control (ASAC) approaches is choosing the appropriate sensor for a given application. While positioning enough microphones in the acoustic field to provide a global estimate of the total sound radiation always provides the necessary error information for controlling the acoustic response of the structure, microphones are often impractical to implement. Since the relationship between the structural response and acoustic response is in general complex, simply minimizing the response of the structure at chosen coordinates does not guarantee minimization of radiated sound, and often times results in an increase in the acoustic response. To alleviate this problem, recent studies have been devoted to developing specialized shaped sensors from polyvinylidene fluoride (PVDF), which respond to the more efficient radiating modes of the structure. ${ }^{1,2}$ Results from these studies in addition to studies where microphones were used as error sensors suggest that the physical mechanism of control can be revealed upon considering a wave-number transform of the structural response. ${ }^{3}$

The wave-number transform, also known as the $k$ transform, is obtained by computing the Fourier transform of the spatial response of the structure. The implications of this approach are well documented by Fahy ${ }^{4}$ and Maidanik. ${ }^{5}$ The wave-number distribution obtained from the transform can be divided into two regions, a supersonic region and a subsonic region. Amplitudes of the wave-number spectrum located in the supersonic region correspond to radiation to the far field while amplitudes in the subsonic region corre- spond to near-field disturbances. Based on this observation, one approach to minimizing the radiated sound of a structure is to minimize the spectrum in the supersonic region of the $k$ transform. In theory this technique can be readily implemented as demonstrated in earlier studies; ${ }^{3,6}$ however, as one might imagine, designing a sensor to measure this part of the response is more difficult.

The thrust of this work is to present a technique whereby measurable quantities can be used as error information to achieve the same result as minimizing the supersonic region of the $k$ transform. This goal is achieved by model reference control with the feedforward filtered- $x$ version of the adaptive LMS algorithm. Accelerometers are utilized to provide the necessary error information, and the complex response of the structure at locations of each accelerometer is computed in advance to determine the appropriate reference response. The desired response at each location on the structure corresponds to that resulting from minimization of the amplitudes in the supersonic region of the $k$ transform. Model reference control can also be applied to complex structures for which analytical solutions of the structural response do not exist. For this case, microphones are used as the initial error sensors, and the resulting structural response after control can be measured at a sufficient number of locations to provide the necessary reference information. The microphones can then be eliminated from the control approach in favor of sensors located on the surface of the structure that yield the same effect.

To demonstrate the model reference control approach, an analytical study was performed with a simply supported beam located in an infinite baffle. The beam is driven har- 
monically with a point force disturbance, and control is achieved with piezoelectric actuators bonded to the surface of the structure. Theory relevant in modeling the structural and acoustical response of the beam will be presented as well as the method of obtaining the $k$ transform. In addition, an overview of the model reference control approach as it pertains to the filtered- $x$ version of the adaptive LMS algorithm will be presented.

\section{THEORY}

To develop the analytical model required in this study, the response of a simply supported beam to a sinusoidal point force disturbance must be obtained as well as the response to a piezoelectric actuator bonded to the surface of the beam. Since the cost function is formulated in terms of the structural response, acoustic response or the wave-number spectrum in the $k$ transform, theory for developing models of each chosen cost function will be presented. In addition to developing equations for the actuators and sensors, a mathematical model of the controller must be developed. For the purpose of this study, linear quadratic optimal control was used to compute the optimal control solution; however, the filtered- $x$ version of the adaptive LMS algorithm is discussed to outline practical implementation of the model reference controller. While lengthy derivations of the equations are omitted from the text, the necessary equations to construct the computer model are included.

\section{A. Disturbance and actuator equations}

As stated earlier, the structure chosen for this particular study is a simply supported beam with the following usual modal response. ${ }^{7}$

$$
w(x, t)=\sum_{m=1}^{\infty} W_{m} \sin \left(\gamma_{m} x\right) \exp (j \omega t),
$$

where $\gamma_{m}=m \pi / L_{x}, W_{m}=$ modal amplitudes, $L_{x}=$ length of beam in $x$ direction, $\omega=$ driving frequency, and $t=$ time.

The response was obtained with a finite summation of the first 10 modes. Based on the frequency range chosen in this study (less than $400 \mathrm{~Hz}$ ) and the resonant characteristics of the structure, 10 modes were more than sufficient for accurately predicting the response of the beam. A schematic of the simply supported beam is presented in Fig. 1 with representative coordinates for all actuators and sensors located on the beam. Solving the equation of motion for the beam subjected to harmonic point force excitation located at a position $c$ on the $x$ axis yields the following expression for the modal amplitudes (including proportional damping):

$$
F_{m}=\frac{2 F \sin \left(\gamma_{m} c\right)}{\rho L_{x}\left(\omega_{m}^{2}-\omega^{2}+j 2 \eta \omega \omega_{m}\right)} .
$$

The resonant frequencies of the simply supported beam expressed in the previous equation are given by

$$
\omega_{m}^{2}=\left(D_{e} / \rho\right)\left(\gamma_{m}\right)^{4},
$$

where the beam flexural stiffness is defined by

$$
D_{e}=E h^{3} w_{b} / 12 \text {, }
$$

and $E=$ Young's modulus of beam, $w_{b}=$ width of beam,

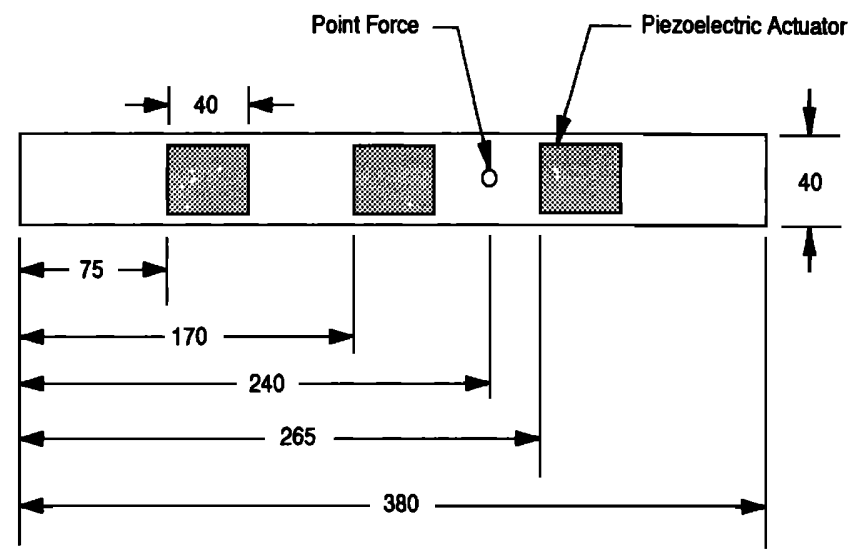

All Dimensions in $\mathrm{mm}$

Beam thickness: $1.96 \mathrm{~mm}$

Piezo thickness: $0.19 \mathrm{~mm}$

FIG. 1. Schematic of simply supported beam.

$h=$ thickness of beam, $\eta=$ damping ratio, and $\rho=$ mass density of beam per unit length.

If we solve the equation of motion for the beam with two piezoelectric patches bonded symmetrically to the front and back surface of the beam and electrically wired out of phase to induce uniform bending about the central axis of the beam, the resulting modal amplitudes can be derived. ${ }^{8}$

$$
\begin{aligned}
P_{m}^{p}= & \frac{2 C_{0} \epsilon_{p e} m \pi}{w_{b} \rho L_{x}^{2}\left(\omega_{m}^{2}-\omega^{2}+j 2 \eta \omega_{m} \omega\right)} \\
& \times\left[\cos \left(\gamma_{m} x_{1}^{p}\right)-\cos \left(\gamma_{m} x_{2}^{p}\right)\right] .
\end{aligned}
$$

Constants used in the above equation are defined as follows.

$$
\begin{aligned}
& \epsilon_{p e}=d_{31} V^{p} / t . \\
& C_{0}=[2 P / 3(1-P)] h^{2} E . \\
& P=-\left(E_{p e} / E\right) K . \\
& K=\frac{6 t h(h+t)}{\left(h^{3}+8 t^{3}\right)+6 h^{2}},
\end{aligned}
$$

where $w_{p e}=$ width of piezoelectric actuator, $d_{31}=$ piezoelectric strain constant, $x_{1}^{p}=x$ coordinates of left corner of $p$ th actuator, $x_{2}^{p}=x$ coordinates of right corner of $p$ th actuator, $V^{p}=$ complex voltage of $p$ th actuator, $t=$ thickness of piezopatch, and $E_{p e}=$ Young's modulus of piezopatch.

To obtain the combined response of excitation by the point force disturbance and piezoelectric actuators, superposition is applied. Based on Eq. (1), the modal amplitudes for the summed response can be expressed as follows:

$$
W_{m}=F_{m}+\sum_{p=1}^{n_{a}} P_{m}^{p},
$$

where $n_{a}=$ number of actuators.

\section{B. Sensor equations}

In the following analysis, error information will be obtained from either of three approaches; the structural response at a point, the acoustic response in the far field at a point, and the supersonic wave-number spectrum of the $k$ transform. In a physical system, an accelerometer or a microphone can be used to obtain error information on the 
structure or in the acoustic field; however, the supersonic region of the $k$ transform is not a directly measurable quantity. To implement a $k$-domain cost function, the structural response of the beam will be used to provide error information, and model reference control will be implemented such that the residual structural response corresponding to the desired wave-number spectrum is obtained. As the LMS algorithm is formulated in the time domain, all error information is measured in the same domain.

\section{Structural error sensor}

If an accelerometer is chosen as the error sensor, an expression for the structural response at the $x$ coordinate of the accelerometer with respect to the beam can be used to develop the cost function modeling the sensor:

$$
\dot{w}(x, t)=\sum_{m=1}^{\infty}-\omega^{2} W_{m} \sin \left(\gamma_{m} x\right) \exp (j \omega t) .
$$

For harmonic excitation, the response of the accelerometer is proportional to the displacement of the structure at the chosen coordinate.

\section{Acoustic error sensor}

If a microphone is chosen as the error sensor, an expression for the pressure can be used in developing the cost function to model the sensor. Rayleigh's integral is used to formulate the expression as a function of the beam response, and numerical techniques can be employed to solve the following equation at the chosen field point: ${ }^{9}$

$$
\begin{aligned}
p\left(x^{\prime}, y^{\prime}, z^{\prime}, t\right)= & -\frac{\omega^{2} \rho_{0}}{2 \pi} \exp (j \omega t) \sum_{m=1}^{\infty} W_{m} \sin \left(\frac{m \pi x}{L_{x}}\right) \\
& \times \int_{0}^{\omega_{b}} \int_{0}^{L_{x}} \frac{\exp \left[-j k \sqrt{\left(x^{\prime}-x\right)^{2}+\left(y^{\prime}-y\right)^{2}+\left(z^{\prime}\right)^{2}}\right]}{\sqrt{\left(x^{\prime}-x\right)^{2}+\left(y^{\prime}-y\right)^{2}+\left(z^{\prime}\right)^{2}}} d x d y,
\end{aligned}
$$

and $\left(x^{\prime}, y^{\prime}, z^{\prime}\right)=$ spatial coordinates of field point, $(x, y, 0)$ $=$ spatial coordinates on beam, $k=$ wave number in acoustic medium, $c=$ speed of sound in acoustic medium, and $\rho_{0}=$ density of acoustic medium.

By replacing $y$ with $y+w_{b} / 2$ and $x+L_{x} / 2$ in Eq. (12) and changing the limits of integration appropriately, the coordinates for computing the pressure are transformed to the center of the beam. A schematic of the coordinate system is presented in Fig. 2.

\section{Wave-number transform}

To implement the $k$ transform of the structural response as a cost function, an expression relating the modal amplitudes of the structural response to the amplitudes of the wavenumber spectrum must be obtained. For those familiar with Fourier transforms of time-dependent signals utilized in signal processing, the $k$ transform (i.e., wave-number transform) is analogous to obtaining the frequency spec-

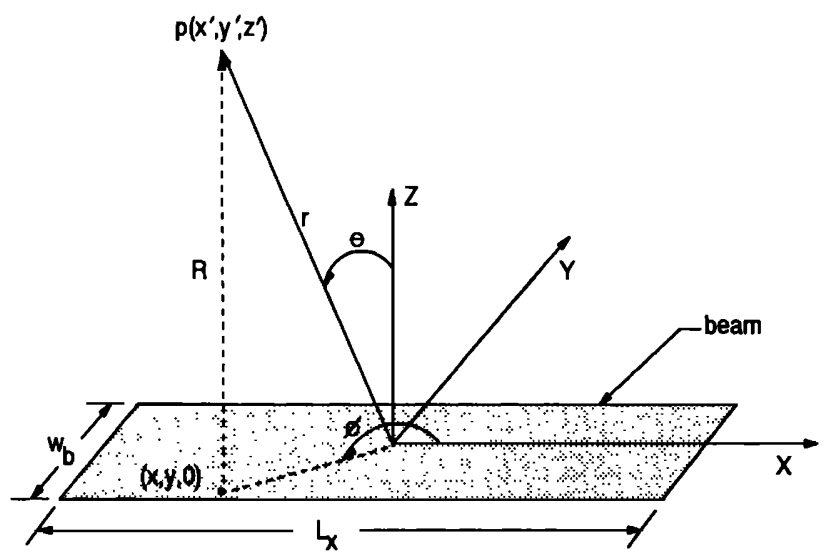

FIG. 2. Coordinate system for pressure radiated from beam. trum from a time-dependent signal. ${ }^{4}$ To obtain the wavenumber transform, a Fourier integral transform of the spatial response is evaluated:

$$
\begin{aligned}
F\left(k_{x}, k_{y}\right)= & \int_{-\infty}^{+\infty} \int_{-\infty}^{+\infty} f(x, y) \exp \left(-j k_{x} x\right) \\
& \times \exp \left(-j k_{y} y\right) d y d x .
\end{aligned}
$$

Replacing $f(x, y)$ with the spatial response of the beam of Eq. (1), and evaluating the integral over the boundaries of the beam (since the baffle out-of-plane response is zero), the wave-number transform is obtained: ${ }^{4}$

$$
\begin{aligned}
W\left(k_{x}, k_{y}\right) & \\
= & \sum_{m=1}^{\infty} W_{m} \frac{\left(m \pi / L_{x}\right)\left[(-1)^{m} \exp \left(-j k_{x} L_{x}\right)-1\right]}{\left[k_{x}^{2}-\left(m \pi / L_{x}\right)^{2}\right]} \\
& \times\left(\frac{\sin \left(k_{y} w_{b} / 2\right)}{k_{y} / 2}\right) .
\end{aligned}
$$

Uncontrolled

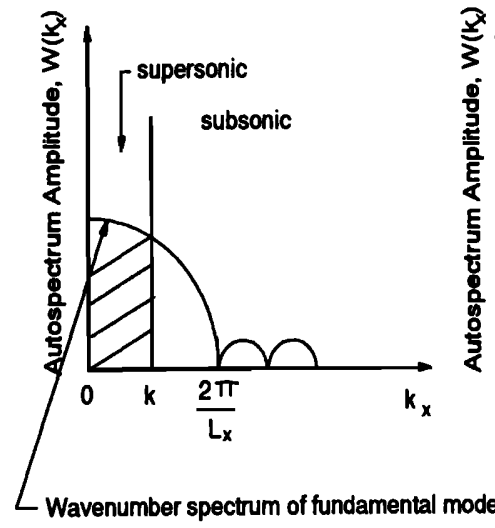

Controlled

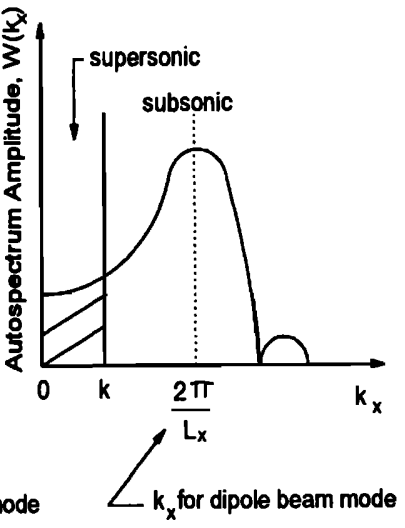

FIG. 3. Example of 1-D $k$ transform (positive components only). 
Plotting the magnitude of this function yields physical insight into the structural acoustic response. An example plot is presented in Fig. 3. The acoustic wave number is defined as follows:

$$
k=\omega / c,
$$

is the critical number defining the region between acoustic waves that radiate to the far field and waves that simply create near-field disturbances. ${ }^{4}$ The radiating region is termed the supersonic region and is the shaded portion in Fig. 3 where the structural wavenumber is less than the acoustic wave number. ${ }^{4}$ The nonradiating region is termed the subsonic region and is defined when the structural wave number is greater than the acoustic wave number. Note that the $k$ transform includes negative values of $k_{x}$ as well, corresponding to radiation at values of $3 \pi / 2>\phi>\pi / 2$ as indicated in Fig. 2.

Using this technique to describe the relationship between the structural and acoustic response of the beam provides a unique method of developing a cost function to minimize the radiated sound from the structure. Although the number of components in the spectrum is obviously infinite, even within the supersonic region, a finite number of spectral amplitudes at particular wave-number values may be chosen for computational purposes. Physically this corresponds to minimizing the sound at corresponding radiation angles. ${ }^{6}$ While this is an esoteric approach for developing a cost function for structural acoustic control, it is physically realizable through model reference control, as detailed below.

\section{Control approach}

\section{Overview of model reference controller}

Before proceeding with the details of the control approach, a brief overview of the model reference controller is in order. A conceptual schematic of a feedforward adaptive model reference controller is presented in Fig. 4(a). The adaptive plant encompasses the structure, control actuators, error sensors, and control dynamics associated with the multi-channel version of the adaptive LMS algorithm, depicted in Fig. 4(b). The reference plant consists of the models required to create the desired response at the chosen error sensor locations, and these models can be obtained analytically or experimentally depending on the designer's choice. The adaptive plant essentially modifies its behavior $[\bar{A} R(k)]$ to achieve the same response as that of the reference plant $[\bar{R}(k)]$, by driving the error $\bar{E}(k)$ to a minimum.

\section{The adaptive LMS algorithm}

The method of control chosen for practical implementation is the multi-channel filtered- $x$ version of the adaptive LMS algorithm. The formulation of this algorithm readily lends itself to model reference control since the disturbance is included in the output of each error sensor. The multichannel version of this algorithm was previously described by Elliot et al., ${ }^{10}$ and will be briefly outlined in this section.

The output of an error sensor can be modeled at the $n$th time step as

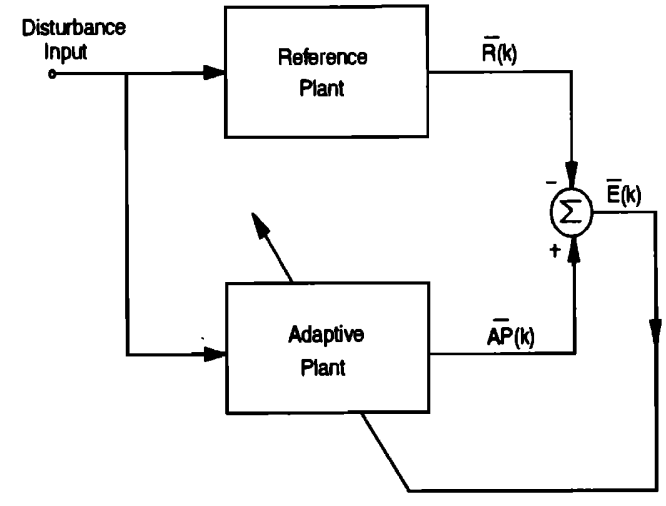

(a) Conceptual Schematic of Model Reference Controller

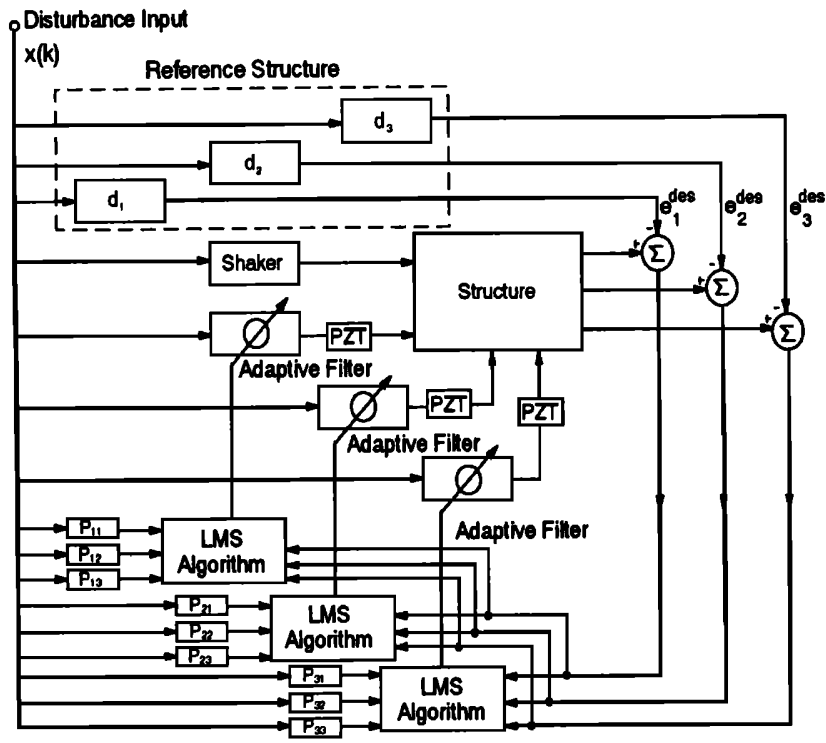

(b) Detailed Schematic of Model Reference Controller

FIG. 4. LMS model reference controller schematic.

$$
\begin{aligned}
e_{l}(n)= & e_{l}^{D}+e_{l}^{\mathrm{des}}+\sum_{m=1}^{M} \sum_{j=0}^{N_{p}-1} P_{l m j} \sum_{i=0}^{N_{w}-1} w_{m i}(n-j) \\
& \times x(n-i-j),
\end{aligned}
$$

where $n=$ time step number, $m=$ actuator number, $l=$ error sensor number, $i=$ adaptive filter coefficient number, $j=$ fixed filter coefficient number, $e_{l}^{D}(n)=$ error due to the disturbance at the $l$ th error sensor, $e_{l}^{\text {des }}(n)=$ desired response at the $l$ th error sensor, $x(n)=$ input reference source, $w_{m i}=$ coefficients of the adaptive finite impulse response (FIR) for the $m$ th actuator and the $i$ th coefficient, $\boldsymbol{P}_{I m j}=j$ th coefficients of the fixed finite impulse response (FIR) between the output of the $m$ th adaptive filter and the $l$ th error sensor, $M=$ number of control actuators, $N_{p}$ $=$ number of fixed filter coefficients, $N_{w}=$ number of adaptive filter coefficients.

In the LMS algorithm, the mean-square error signal is 
defined by

$$
J=E\left(\sum_{l=1}^{L} e_{l}^{2}(n)\right),
$$

where $E$ is the expectation operator. Since this error function is quadratic with respect to the filter coefficients, only one minimum solution exists. The outputs of the fixed filters, $P_{l m j}$, at each time step $n$, were used by the LMS algorithm to minimize the mean-square error signal by modifying the coefficients of the adaptive filter as follows:

$$
w_{m i}(n+1)=w_{m i}(n)-\mu \sum_{l=1}^{L} e_{l}(n) r_{l m}(n-i)
$$

and

$$
r_{l m}(n-i)=\sum_{j=0}^{N_{p}-1} P_{l m j} x(n-i-j) .
$$

In achieving control, the adaptive finite impulse response filter coefficients converge to a steady state value. In typical applications, these coefficients, when multiplied by the reference signal, yield a response equal in magnitude and opposite in sign to the disturbance present (when the number of independent actuators equals the number of error sensors), driving the response at the chosen error sensor to some minimum value. For the case of model reference control, the desired response at the $l$ th error sensor is no longer zero, but rather some predetermined system response.

To implement the equations modeling the error sensors and actuators in the LMS algorithm, the weighting coefficients in the equations for the response of the error sensors must be determined. A schematic of the control approach is presented in Fig. 4(b). The coefficients $P_{l m j}$ define the transfer functions between the control actuators and the error sensors chosen. For modeling purposes, the input voltage to the actuator is set equal to 1 in Eq. (5), and the response from each error sensor of Eq. (11), (12), or (16) is computed based on modal amplitudes obtained from Eq. (5). Upon substituting the modal amplitudes in the expression for the chosen error sensor, the necessary transfer function is obtained in the frequency domain.

The desired response for model reference control is generated from an FIR with coefficients defined by $d_{l}$, as depicted in Fig. 4(b). ${ }^{11}$ For example, the output of the filter $d_{1}$ can be computed for a harmonic signal with two coefficients as follows:

$$
e_{1}^{\text {des }}(k)=d_{1_{0}} x(k)+d_{1_{1}} x(k-1) .
$$

If the desired response at each error sensor is zero, the coefficients defined by $d_{l}$ are simply set equal to zero. As the adaptive coefficients begin to converge, the error signal is driven to a minimum, and hence the response is driven to the desired value at each error sensor. Upon converging, the adaptive coefficients $w_{m i}$ contain the necessary information for computing the optimal control voltage to each actuator. While the previous development has been limited to a single frequency, the multi-frequency control case can be developed by simply including two additional coefficients for each frequency present. In broadband applications, an FIR may be insufficient for modeling the system, and Vipperman $e t$ $a l$. , have demonstrated that an infinite impulse response
(IIR) filter can be implemented in this case. ${ }^{12}$

Before executing the LMS algorithm, the frequency domain transfer functions must be converted to time domain coefficients in the form of finite impulse response filter weights. Upon achieving control, it is also convenient to convert the converged adaptive weights back to the frequency domain to ascertain magnitude and phase information. This transformation of time domain coefficients to the frequency domain and vice versa is easily achieved with simple trigonometry. For a sinusoidal input, two coefficients are required to model the signal. In this case, the transformation can be represented by the following equations:

$$
\operatorname{Re}\left(V_{m}\right)=w_{m 1}+w_{m 2} \cos (\omega \Delta t)
$$

and

$$
\operatorname{Im}\left(V_{m}\right)=-w_{m 2} \sin (\omega \Delta t),
$$

where $\Delta t=$ time between discrete samples, $\operatorname{Im}\left(V_{m}\right)$ $=$ imaginary part of the complex voltage, and $\operatorname{Re}\left(V_{m}\right)$ $=$ real part of the complex voltage.

\section{Linear quadratic optimal control}

While the previously outlined control approach was modeled and will be implemented in hardware in the future, linear quadratic optimal control theory was utilized to compute the optimal control voltages for the present study. These two control approaches were observed to yield the same optimal solution in a previous study by the authors. ${ }^{3}$ For the problem at hand, the supersonic region of the wavenumber transform or error microphones are chosen as a cost function, and the function is minimized by adjusting the voltage at each piezoelectric actuator appropriately. As discussed in previous work, ${ }^{3}$ the cost function can be formulated as a sum of the squares:

$$
C=\sum_{b=1}^{n_{e}} q_{b} q_{b}^{*}
$$

where $q_{b}=$ output of error sensor, $q_{b}^{*}=$ complex conjugate of $q_{b}$, and $n_{e}=$ number of error sensors.

The error sensor output can be formulated in terms of the transfer functions between the sensors and both the disturbance and the control actuators as follows:

$$
q_{b}=\left(\sum_{s=1}^{n_{a}} T_{b s} \hat{V}_{s}\right)+T_{b D} F,
$$

where $T_{b s}=$ transfer function between sth actuator and $b$ th error sensor, $T_{b D}=$ transfer function between the disturbance and $b$ th error sensor, $\widehat{V}_{s}=$ complex voltage of $s$ th actuator, $F=$ input force, and $n_{a}=$ number of control actuators.

The transfer functions listed above are not to be viewed as specific to any particular type of error sensor. For example, if the structural response is taken as an error signal, the transfer function between the chosen error sensor and the disturbance can be constructed as follows:

$$
T_{b D}^{a}=-\omega^{2} \sum_{m=1}^{M} \frac{F_{m}}{F} \sin \left(\gamma_{m} x_{b}\right) .
$$

Similarly, the same transfer function can be created for the resulting radiated pressure, or a spectral line of the $k$ trans- 
form, respectively, as follows:

$$
T_{b D}^{p}=-\frac{\omega^{2} \rho_{0}}{2 \pi} \sum_{m=1}^{M} \frac{F_{m}}{F} \sin \left(\frac{m \pi x_{b}}{L_{x}}\right) \int_{0}^{w_{b}} \int_{0}^{L_{x}} \frac{\exp \left[-j k \sqrt{\left(x^{\prime}-x_{b}\right)^{2}+\left(y^{\prime}-y_{b}\right)^{2}+\left(z^{\prime}\right)^{2}}\right]}{\sqrt{\left(x^{\prime}-x_{b}\right)^{2}+\left(y^{\prime}-y_{b}\right)^{2}+\left(z^{\prime}\right)^{2}}} d x d y
$$

$$
\begin{aligned}
T_{b D}^{k}= & \sum_{m=1}^{M} \frac{F_{m}}{F} \frac{\left(m \pi / L_{x}\right)\left[(-1)^{m} \exp \left(-j k_{x_{b}} L_{x}\right)-1\right]}{\left[k_{x_{b}}^{2}-\left(m \pi / L_{x}\right)^{2}\right]} \\
& \times\left(\frac{\sin \left(k_{y_{b}} w_{b} / 2\right)}{k_{y b} / 2}\right)
\end{aligned}
$$

The transfer functions for the control actuators $T_{b s}$ can be constructed from Eqs. (25), (26), and (27) by simply replacing the force $F$ with the applied voltage $\hat{V}$, and the modal amplitudes corresponding to point force excitation $F_{m}$ with the modal amplitudes corresponding to excitation by the $p$ th piezoceramic actuator $P_{m}^{p}$.

Proceeding with the solution, taking the partial derivative of the cost function with respect to the real and imaginary part of each control voltage results in the following system of linear equations that must be solved:

$\left[\sum_{b=1}^{n_{e}}\left(\sum_{l=1}^{n_{a}} T_{b l} \widehat{V}_{l}+T_{b D} F\right) T_{b s}^{*}=0\right], \quad s=1, n_{a}$.

The previous set of equations can be represented with linear algebra

$$
[A][\hat{V}]=[b] .
$$

Since the number of unknowns is equivalent to the number of equations, the solution is obtained as follows:

$$
[\hat{V}]=[A]^{-1}[b],
$$

for a nonsingular matrix $[A]$.

\section{Model reference approach}

To include a reference response in the model, the cost function defined in Eq. (23) must be modified. As opposed to minimizing the response at the coordinates of a chosen error sensor, the difference between the response at that error sensor and the desired response is minimized. In other words, the response at the error sensor is driven to the model reference response. The cost function presented in Eq. (23) is thus modified as follows:

$$
C_{\mathrm{ref}}=\sum_{b=1}^{n_{e}}\left[q_{b}-\widehat{\operatorname{ref}}\left(x_{b}\right)\right]\left[q_{b}-\widehat{\operatorname{ref}}\left(x_{b}\right)\right]^{*},
$$

where $\widehat{\operatorname{ref}}\left(x_{b}\right)$ is the desired response at the $b$ th error sensor. The system of linear equations presented in Eq. (28) can be modified to include the reference response as follows:

$$
\left[\sum_{b=1}^{n_{e}}\left(\sum_{l=1}^{n_{a}} T_{b l} \hat{V}_{l}+T_{b D} F-\widehat{\operatorname{ref}}\left(x_{b}\right)\right) T_{b s}^{*}=0\right], \quad s=1, n_{a} .
$$

Obviously if the reference response, $\widehat{\operatorname{ref}}\left(x_{b}\right)$, is set equal to zero, the above expression reduces to Eq. (28).

Model reference control yields a unique method of implementing a $k$-domain cost function or replacing error sensors such as microphones with more favorable structural sensors. To determine the appropriate reference structural response, the cost function is originally formulated as in Eq. (23), using either the radiated pressure at chosen coordinates or corresponding spectral lines of the $k$ transform as the error information. The optimal solution is then obtained by either using Eq. (30) or, in practice, minimizing with the adaptive filtered- $x$ LMS algorithm using Eq. (16) for the error signal with the desired response set to zero. The corresponding residual (controlled) beam complex response is then either calculated or, in practice, measured at the required points. The control approach is then switched to structural sensors (e.g., accelerometers) at the same reference points in conjunction with the required reference model. Analytically, the reference model is implemented using a shifted cost function as defined in Eq. (31). In practice, the reference model is implemented by passing the disturbance signal through fixed finite impulse response filters, whose coefficients are defined by $d_{l}$ and can be computed from standard system identification approaches. The adaptive filtered- $x$ LMS algorithm is then implemented to minimize the error signal(s) defined by Eq. (16) in which the desired response is now given by the output of the fixed model reference FIR filters.

As demonstrated in the Appendix, when the number of reference sensors is at least equal to the number of control actuators, then the reference motion of the beam is uniquely defined using the model reference approach. This result is very useful since the original cost function can be formulated with as many error sensors as desired; however, the number of error sensors resulting in the desired control condition can be reduced to a number of structural sensors equivalent to the number of control actuators implemented. Hence model reference control can be implemented to achieve the identical control condition previously obtained from the $k$-domain error information, including as many spectral lines in the cost function as desired or the pressure error information, including as many sensor locations as desired. The cost function is then constructed from the residual structural response at a number of coordinates at least equivalent to the number of control actuators, and the response is driven to the predetermined value at each coordinate on the beam. Thus for example, if global sound control is achieved with one actuator and three acoustic sensors, then only a single structural reference point is required to achieve the same performance as demonstrated in the Appendix, thereby reducing the number of inputs to the controller.

\section{BEAM CONSTRAINTS AND CONFIGURATION}

For the purpose of this study, the simply supported beam was assumed to be located in an infinite baffle. The beam model includes material properties for steel, and the dimensions were chosen such that the beam measured 380 $\mathrm{mm}$ long $\times 40 \mathrm{~mm}$ wide $\times 1.96 \mathrm{~mm}$ thick. Since the re- 
TABLE I. Theoretical resonant frequencies for simply supported beam.

\begin{tabular}{cc}
\hline \hline Mode & Theoretical $f_{n}(\mathrm{~Hz})$ \\
\hline 1 & 31 \\
2 & 124 \\
3 & 279 \\
4 & 495 \\
5 & 774 \\
6 & 1115 \\
7 & 1517 \\
8 & 1982 \\
9 & 2509 \\
10 & 3100 \\
\hline \hline
\end{tabular}

sponse of the structure is assumed one dimensional, all coordinates for locating sensors and actuators will be specified with reference to the $x$ dimension only. All theoretical studies will be conducted with the point force disturbance located at $240 \mathrm{~mm}$ from the end as depicted in Fig. 1. For all test cases conducted, the input force was set equal to $1 N$. Three piezoelectric actuators, $40 \mathrm{~mm}$ long $\times 30 \mathrm{~mm}$ wide $\times 0.19$ $\mathrm{mm}$ thick, were located on the beam as illustrated in Fig. 1 . The material properties of the piezoceramic actuators modeled in this study were based on those tabulated in the "Piezoelectric Motor/Actuator Kit Manual" supplied by Piezo Systems for G1195. ${ }^{13}$

Before presenting results, the resonant frequencies of the simply supported beam for the chosen dimensions and material properties are presented. The resonant frequencies documented in Table I enable the reader to determine modes of interest for the chosen off-resonance test cases (which are typically the most difficult cases to control) in the following summary of the results.

\section{RESULTS}

Two analytical test cases were chosen to demonstrate potential applications for model reference control with the feedforward filtered-x LMS algorithm. In the first case, the acoustic pressure evaluated at discrete field points was chosen to construct the cost function, and in the second case, the supersonic region on the $k_{x}$ axis of the wave-number domain was chosen as the cost function. In both cases, the resulting controlled structural response was computed at chosen coordinates on the beam to provide the necessary response desired in the model reference control approach. In the final section, a sensitivity analysis was performed to determine the level of control achieved for phase and magnitude errors in the models used to compute the desired response as well as variation in frequency of excitation.

\section{A. Model reference control based on a microphone cost function}

For the first test case, a driving frequency of $400 \mathrm{~Hz}$ was chosen for the disturbance, corresponding to an off-resonance condition for the simply supported beam as indicated in Table I. The cost function was constructed from the computed acoustic response of the structure at the following three field points: $\left(r=1.2 \mathrm{~m}, \theta=60^{\circ}, \phi=0^{\circ}\right),(r=1.2 \mathrm{~m}$, $\left.\theta=0^{\circ}, \phi=0^{\circ}\right)$ and $\left(r=1.2 \mathrm{~m}, \theta=60^{\circ}, \phi=180^{\circ}\right)$ which are indicated in Fig. $5(\mathrm{a})$ as $60^{\circ}, 0^{\circ}$, and $-60^{\circ}$, respectively. Upon achieving control, the phase-referenced structural response at three axial locations on the beam of $84.4,126.7$, and $168.9 \mathrm{~mm}$ was computed. To implement model reference control, the structural response was chosen as the cost function, and the computed reference structural response was included in the model as outlined earlier. Both the farfield acoustic directivity pattern and spectral amplitudes of the wave-number transform of the structure are presented graphically in Fig. 5(a) and (b), respectively. The far-field acoustic directivity pattern is plotted along the $x$ axis of the plate with respect to Fig. 2 (i.e., $\phi=0^{\circ}, 180^{\circ}$ ). For convenience, negative values of $\theta$ correspond to $\phi=180^{\circ}$.

For comparison, the structural response was driven to zero at the above chosen coordinates to emphasize the difference between model reference control and simply minimizing the structural response. As is shown in Fig. 5(a), the

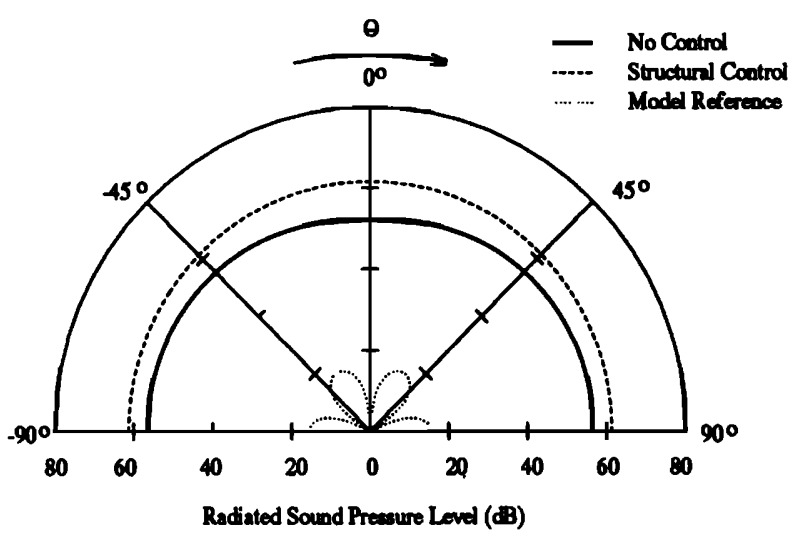

(a) Radiation Directivity

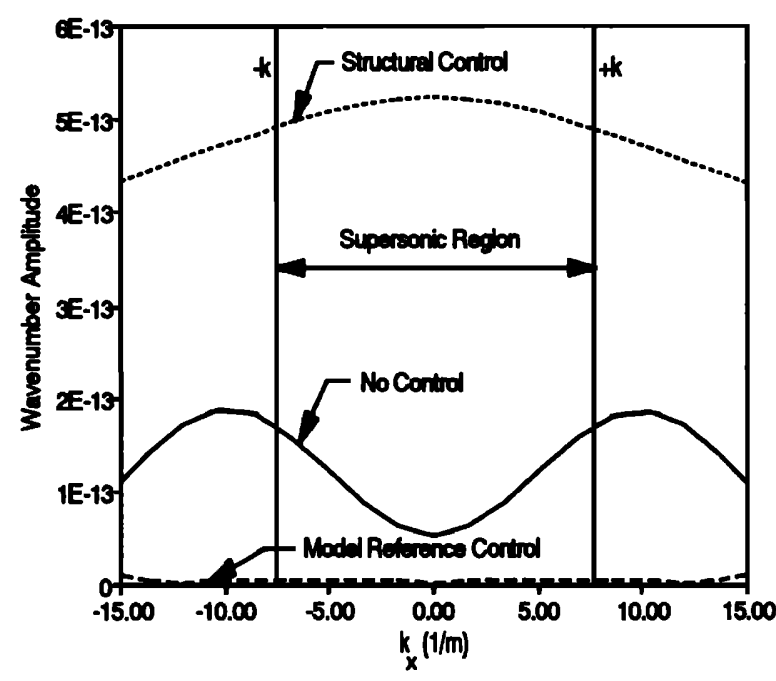

(b) Wavenumber Distribution

FIG. 5. Model reference control implemented from microphone error data, $f=400 \mathrm{~Hz}$ 
acoustic response actually increases when the structural response is simply minimized at the chosen coordinates; however, if the response is driven to the computed model reference response corresponding to the original acoustic cost function based on the sound pressure at the three chosen field points, the acoustic response is attenuated by approximately $40 \mathrm{~dB}$. The physical mechanism of control is emphasized in the plot of Fig. 5(b) comparing the wave-number transform. In the model reference control approach, the wave-number spectrum is minimized significantly in the supersonic region (where $-7.4 \mathrm{~m}^{-1}<k_{x}<+7.4 \mathrm{~m}^{-1}$ ). When the structural response is simply minimized, the opposite occurs, and the amplitudes of the wave-number spectrum in the supersonic region actually increase. In a physical system where permanently locating microphones in the radiated field may not be convenient, a model reference control approach can be implemented, replacing the acoustic transducers with more favorable structural transducers.

\section{B. Model reference control based on $\boldsymbol{k}$-domain cost function}

As in the previous test case, a driving frequency of 400 $\mathrm{Hz}$ was chosen for the disturbance. The supersonic region of the $k$-transform for this case lies between $-7.4 \mathrm{~m}^{-1}$ and $+7.4 \mathrm{~m}^{-1}$ on the $k_{x}$ axis. To emphasize the possibilities afforded with a model reference control approach, the cost function was constructed from seven equally spaced spectral lines lying within the supersonic region of the wave-number spectrum along the $k_{x}$ axis depicted in Fig. 6(b). In effect, complex wave-number information in each of these ranges becomes the error signal. As in the previous test case, the residual structural response was computed at the same three coordinates upon achieving control with the $k$-domain cost function. This computed response was implemented in the model reference control as the desired structural response. As indicated in Fig. 6(a), the global far-field sound radiation from the beam was attenuated by approximately 35 $\mathrm{dB}$. In addition, the amplitudes of the wave-number spectrum were reduced significantly in the supersonic region as observed in Fig. 6(b).

\section{Sensitivity analysis of model reference control}

A sensitivity analysis was performed to determine the impact of inaccuracies of the models (i.e., inaccuracy in the model reference structure) used to obtain the desired response on the level of control achieved. This is not intended as a "robustness" analysis as this is beyond the scope of this paper; however, the following analysis gives the designer an idea of the change in response characteristics of the system due to errors in system modeling. In addition, the frequency of excitation was perturbed by a small amount to determine the effect of fluctuations in the reference frequency.

In a practical controller, implemented with the filtered$x$ version of the adaptive LMS algorithm, errors in modeling the FIR filters necessary to create the reference response can occur. To study the effect of this error, the transfer functions used to compute the reference response for the linear quadratic optimal control approach were initially perturbed

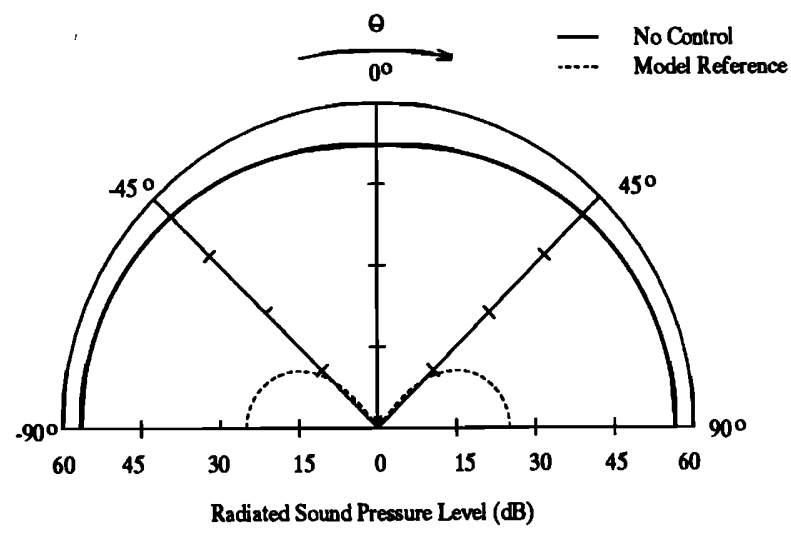

(a) Radiation Directivity

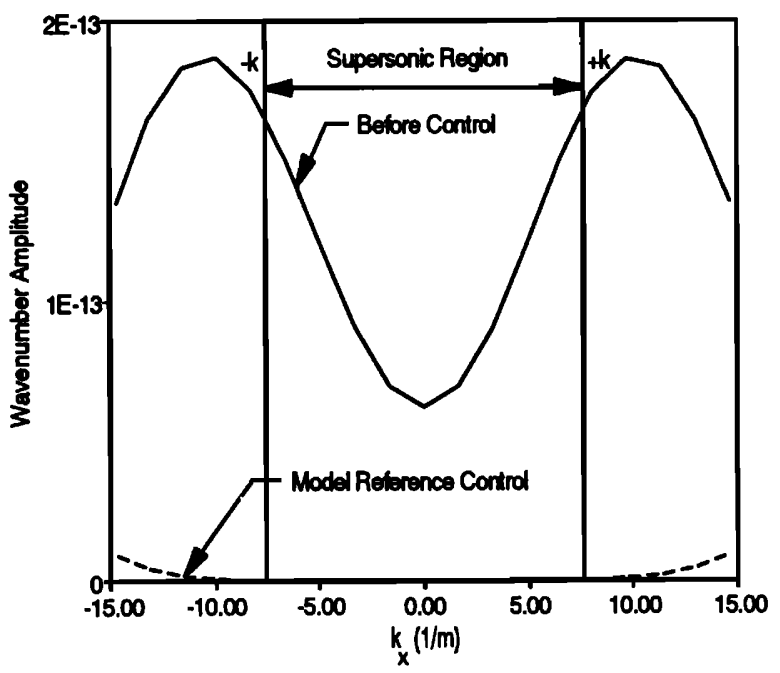

(b) Wavenumber Distribution

FIG. 6. Model reference control implemented from wave-number tranform error data, $f=400 \mathrm{~Hz}$

$\pm 5 \%$ in magnitude (from the perspective of a typical frequency response function) to determine the effect on the level of sound attenuation achieved. As illustrated in Fig. 7 (a), approximately $20 \mathrm{~dB}$ of attenuation in far-field sound radiation was achieved for both cases. Observe that the optimal solution for the model reference controller resulted in approximately $40 \mathrm{~dB}$ of attenuation in sound in the residual acoustic field (i.e., at locations other than that of the chosen acoustic field points). Errors in the magnitude of the transfer functions affect the global control achieved; however, attenuation in the residual acoustic field is only $20 \mathrm{~dB}$ less than that achieved when the optimal solution is obtained. It also should be noted that $\mathbf{4 0} \mathrm{dB}$ of attenuation is approximately the limit of practical adaptive control systems with present hardware.

As a second case, a $\pm 5.0^{\circ}$ phase error (from the perspective of a typical frequency response function) was introduced on all of the transfer functions necessary to create the desired response to evaluate the effect on the level of acoustic 


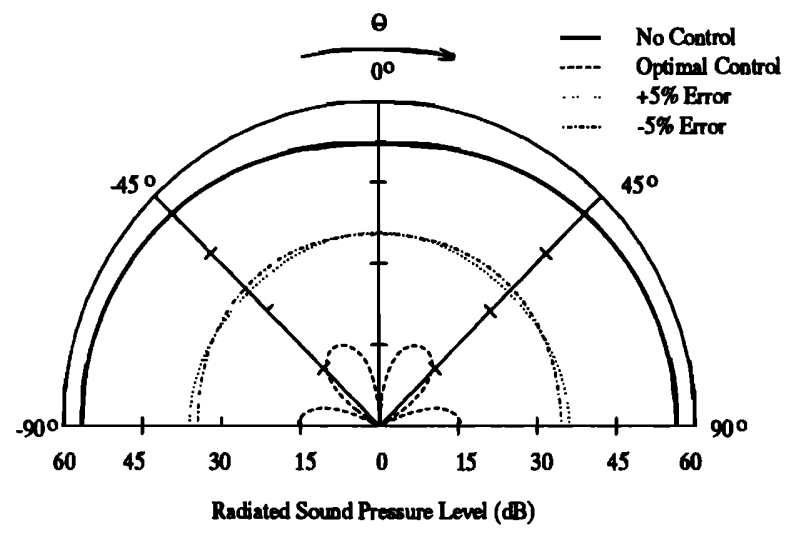

(a) Error in Magnitude

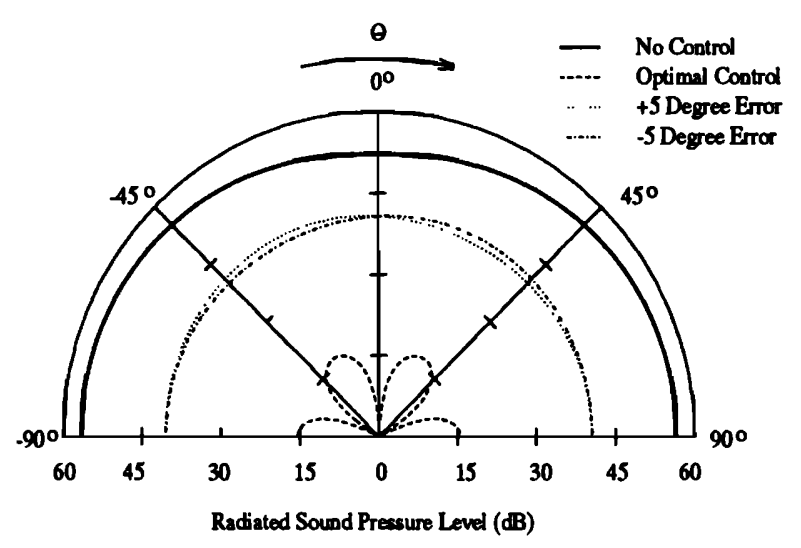

(b) Error in Phase

FIG. 7. Model reference control implemented from microphone error data, $f=400 \mathrm{~Hz}$.

attenuation obtained. The results are plotted in Fig. 7(b), and as can be seen, the levels of attenuation were on the order of $15 \mathrm{~dB}$. This corresponds to a loss in the acoustic attenuation in the residual field of approximately $25 \mathrm{~dB}$. In many practical applications, this may be an acceptable compromise when considering the advantages afforded by replacing the microphone error sensors with accelerometers located on the surface of the structure. In addition, $\pm 5.0^{\circ}$ error in phase is a significant level to impose on the reference model.

In the final case of the sensitivity analysis, the frequency of excitation was shifted by $\pm 5 \%$; however, the model for the reference response was left at that determined for an excitation frequency of $400 \mathrm{~Hz}$. The results presented in Fig. 8 (a) correspond to a reference frequency of $420 \mathrm{~Hz}$ while the results depicted in Fig. 8(b) correspond to a reference frequency of $380 \mathrm{~Hz}$. Unlike the previous set of results, different plots were required for each case since the uncontrolled acoustic response will obviously differ with excitation frequency. For both cases, the acoustic attenuation

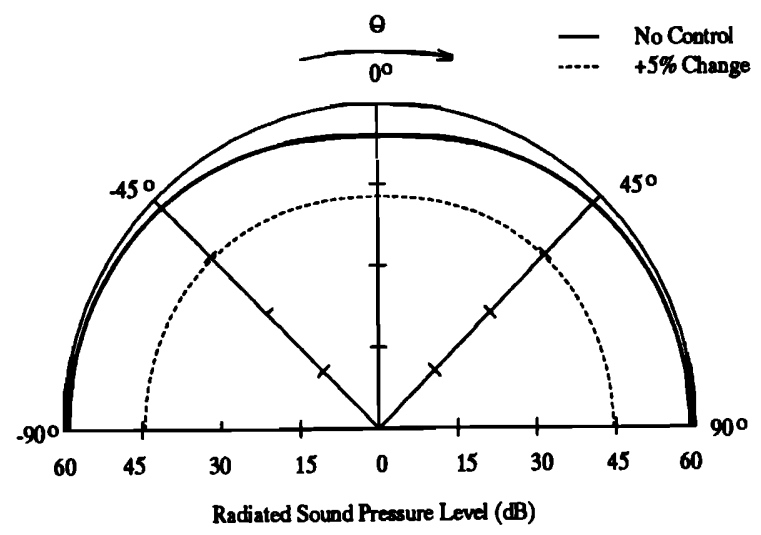

(a) Reference Frequency of $380 \mathrm{~Hz}$

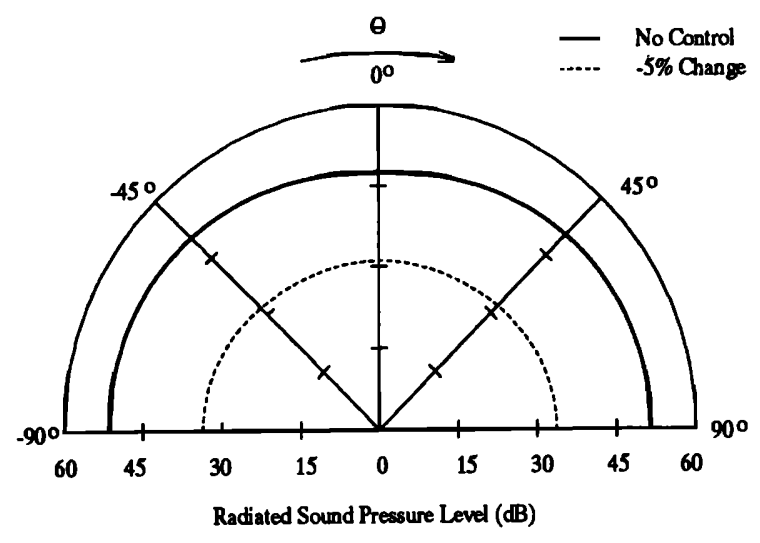

(b) Reference Frequency of $420 \mathrm{~Hz}$

FIG. 8. Model reference control implemented from microphone error data with shift in reference frequency.

achieved in the residual field was approximately $15 \mathrm{~dB}$. This level of attenuation was observed from approximately 350 to $450 \mathrm{~Hz}$. In general, the relative phasing between the accelerometers implemented in the model reference control approach demand a certain phasing between modes present on the structure. While this may not corresond to the optimal control obtained when microphones are implemented as error sensors, a reasonable level of acoustic attenuation is attainable.

In effect, the structural sensors (i.e., accelerometers) implemented in the model reference controller can be viewed as a distributed cost function, demanding a required phase relationship at various axial locations on the structure. This relationship can be met only if the desired phasing between relative modes is obtained. Since the original cost function was formulated in the acoustic field, the phasing between modes corresponds to that resulting in minimum sound radiation upon achieving control. This observation is valid for cases such as that presented where the modal density is low 
and only a few modes contribute significantly to the structural and acoustic response. For cases exhibiting higher modal densities, the level of attenuation possible deviates more significantly as the frequency of excitation is shifted from that used in determining the models required to create the desired response. This technique is ideally suited for structural acoustic control since the excitation frequency is generally low, and the response is dominated by a limited number of modes. A good rule of thumb is to implement a number of structural sensors in the model reference control approach equivalent to the number of dominant modes in the acoustic response. While this is not necessary to achieve the optimal control condition (recall that the number of structural sensors must simply equal the number of control actuators), reasonable levels of far-field sound attenuation can be achieved for significant deviations in the reference frequency.

\section{CONCLUSIONS}

Results from this study demonstrate that model reference control is a valid method of replacing cost functions based on acoustic sensors such as microphones with more favorable structural sensors such as accelerometers. In addition, the model reference control implementation provides a unique method of using structural error sensors to create a wave-number domain controller. In either case, the number of structural error sensors required to duplicate the desired controlled structural acoustic response must simply equal the number of control actuators implemented as long as the transfer functions between the sensors and actuators are unique.

Results from the sensitivity analysis indicate that deviation in magnitude on the order of $\pm 5 \%$ for the transfer functions used to create the desired reference response results in attenuation of far-field sound radiation in the residual field of approximately $20 \mathrm{~dB}$. In addition, a $\pm 5.0^{\circ}$ perturbation of the phase of the transfer functions used to create the reference response resulted in approximately $15 \mathrm{~dB}$ of attenuation in sound radiation. In the final part of the sensitivity analysis, the reference frequency was shifted by $\pm 5 \%$, and the level of acoustic attenuation observed in the radiated field was approximately $15 \mathrm{~dB}$. While the reference plant was designed for a specific test frequency $(400 \mathrm{~Hz}$ in this case), significant levels of sound attenuation were predicted when operating at frequencies other than the design frequency.

Although predicted levels of acoustic attenuation resulting from modeling errors in the reference plant as well as variations in input frequency are obviously less than that obtained under ideal operating conditions, the level of acoustic attenuation may be acceptable in many cases considering advantages afforded by placing the error sensors on the structure as opposed to locating them in the radiated acoustic field. Many physical systems are based on stationary signals for which the acoustic field remains relatively unchanged, and for these systems model reference control provides a unique method of achieving the desired acoustic response without implementing microphones as error sensors.

\section{ACKNOWLEDGMENT}

This work was supported by DARPA/ONR Grant No. ONR-N00014-88-K-0721.

\section{APPENDIX: MODEL REFERENCE EXAMPLE}

Model reference control can be implemented to yield the desired structural acoustic response of the system by simply driving the structure to a reference response corresponding to the desired acoustic directivity pattern. If the transfer functions between the control actuators implemented and the chosen structural error sensors are unique, then the number of structural sensors required to achieve the desired controlled acoustic response need only be equal to the number of control actuators implemented. Thus, while the original cost function can be based on any number of acoustic error sensors, the number of structural sensors must simply equal the number of control actuators required to achieve control. An example is provided whereby the original cost function is formulated in terms of the acoustic response of the beam, and three unique acoustic field points are chosen to minimize the response. To simplify the example, only one control actuator is used to achieve control. This cost function is replaced by a model reference cost function implementing only one structural error sensor. The controlled response is shown to be unique, and this can readily be extended to the general case. Referring to Eq. (28), an expression for the optimal control voltage is obtained in terms of the transfer functions between the three error sensors and the control actuator as well as the disturbance:

$$
\begin{aligned}
& \left(T_{11} T_{11}^{*}+T_{21} T_{21}^{*}+T_{31} T_{31}^{*}\right)^{p} \widehat{V}_{1}^{p} \\
& \quad+\left(T_{1 D} T_{11}^{*}+T_{2 D} T_{21}^{*}+T_{3 D} T_{31}^{*}\right)^{p} F=0 .
\end{aligned}
$$

In the above equation, the superscript $p$ is used to denote that the transfer functions were formulated in terms of pressure as outlined in Eq. (26). The optimal control voltage can be obtained as follow:

$$
\widehat{V}_{1}^{p}=-\left(\frac{T_{1 D} T_{11}^{*}+T_{2 D} T_{21}^{*}+T_{3 D} T_{31}^{*}}{T_{11} T_{11}^{*}+T_{21} T_{21}^{*}+T_{31} T_{31}^{*}}\right)^{p} F
$$

With this optimal control voltage for the piezoceramic actuator, the modal amplitudes for the structural response can be obtained as outlined in Eqs. (5) and (10):

$$
W_{m}=F_{m}+P_{m}^{1 p} .
$$

Upon obtaining the above modal amplitudes, the necessary information required to compute the reference response results. Since only one control actuator is used to obtain the desired acoustic response, only one structural sensor is required in the model reference approach to obtain the same controlled response. The cost function presented in Eq. (31) is now formulated in terms of the structural response where the desired response at any arbitrary coordinate on the beam is expressed as follows:

$$
\widehat{\operatorname{ref}}\left(x_{1}\right)=\sum_{m=1}^{M}\left(F_{m}+P_{m}^{1 p}\right) \sin \left(\gamma_{m} z_{1}\right) \text {. }
$$

Substituting Eq. (A4) into Eq. (32) yields: 


$$
\begin{aligned}
& \left(T_{11} T_{11}^{*}\right)^{a} \widehat{V}_{1}^{a}+\left(T_{1 D} T_{11}^{*}\right)^{a} F \\
& \quad-\left(\sum_{m=1}^{M}\left(F_{m}+P_{m}^{1 p}\right) \sin \left(\gamma_{m} x_{1}\right)\right) T_{11}^{*}=0
\end{aligned}
$$

where the superscript $a$ is used to differentiate the transfer functions and control voltage for the structural response from the transfer functions and control voltage previously defined in terms of the pressure. The transfer function between the structural response at coordinate $x_{1}$ and the input force, $F$, can be expressed as below:

$$
T_{1 D}^{a}=\frac{1}{F} \sum_{m=1}^{M} F_{m} \sin \left(\gamma_{m} x_{1}\right)
$$

Substituting Eq. (38) into Eq. (37), we obtain

$T_{11}^{a} \hat{V}_{1}^{a}+T_{1 D}^{a} F-\left(T_{1 D}^{a} F+\sum_{m=1}^{M} P_{m}^{1 p} \sin \left(\gamma_{m} x_{1}\right)\right)=0$.

The expression for the modal amplitudes was given in Eq. (5) as a function of the voltage applied to the piezoelectric actuator. For simplicity, all constants in Eq. (5) will be represented by $K_{m}^{1}$, and the modal amplitudes resulting from the optimal voltage obtained for minimization of the pressure can be expressed as follows:

$$
\begin{aligned}
P_{m}^{1 p} & =K_{m}^{1} \widehat{V}_{1}^{p} \\
& =-K_{m}^{1}\left(\frac{T_{1 D} T_{11}^{*}+T_{2 D} T_{21}^{*}+T_{3 D} T_{31}^{*}}{T_{11} T_{11}^{*}+T_{21} T_{21}^{*}+T_{31} T_{31}^{*}}\right)^{p} F .
\end{aligned}
$$

Substituting Eq. (A8) into Eq. (A7), and canceling like terms, we see that the optimal voltage obtained from the model reference control approach is a function of the transfer functions for the far-field pressure:

$$
\begin{aligned}
T_{11}^{a} \hat{V}_{1}^{a}= & -\sum_{m=1}^{M} K_{m}^{1}\left(\frac{T_{1 D} T_{11}^{*}+T_{2 D} T_{21}^{*}+T_{3 D} T_{31}^{*}}{T_{11} T_{11}^{*}+T_{21} T_{21}^{*}+T_{31} T_{31}^{*}}\right)^{p} \\
& \times F \sin \left(\gamma_{m} x_{1}\right) .
\end{aligned}
$$

The transfer function between the structural response at coordinate $x_{1}$ and the input voltage can be simply expressed as:

$$
T_{11}^{a}=\sum_{m=1}^{M} \frac{P_{m}^{1 a}}{\widehat{V}_{1}^{a}} \sin \left(\gamma_{m} x_{1}\right)
$$

Substituting Eq. (A10) into Eq. (A9) and cancelling like terms, the modal amplitudes resulting from model reference control can be expressed simply as a function of the acoustic transfer functions:

$$
\begin{aligned}
\sum_{m=1}^{M} P_{m}^{1 a} \sin \left(\gamma_{m} x_{1}\right) \\
=-\sum_{m=1}^{M} K_{m}^{1}\left(\frac{T_{1 D} T_{11}^{*}+T_{2 D} T_{21}^{*}+T_{3 D} T_{31}^{*}}{T_{11} T_{11}^{*}+T_{21} T_{21}^{*}+T_{31} T_{31}^{*}}\right)^{p} \\
\quad \times F \sin \left(\gamma_{m} x_{1}\right) ;
\end{aligned}
$$

therefore,

$$
P_{m}^{1 a}=-K_{m}^{1}\left(\frac{T_{1 D} T_{11}^{*}+T_{2 D} T_{21}^{*}+T_{3 D} T_{31}^{*}}{T_{11} T_{11}^{*}+T_{21} T_{21}^{*}+T_{31} T_{31}^{*}}\right)^{p} F
$$

Since the modal amplitudes resulting from model reference control implementing a single structural sensor are identical to those obtained by minimizing the far-field pressure at three error sensor locations, the resulting control voltage applied to the piezoceramic actuator must be the same as that obtained when implementing the acoustic cost function. The previous example can readily be generalized for an arbitrary number of actuators and error sensors as long as the number of error sensors is at least equivalent to the number of control actuators.

${ }^{1}$ R. L. Clark and C. R. Fuller, "Control of sound radiation with adaptive structures," J. Intelligent Mater. Systems Structures 2(3), 431-452 (1991).

${ }^{2}$ R. L. Clark and C. R. Fuller, "Modal sensing of efficient acoustic radiators with PVDF distributed sensors in active structural acoustic approaches," J. Acoust. Soc. Am. 91, 3321-3329 (1992).

${ }^{3}$ R. L. Clark and C. R. Fuller, "Active structural acoustic control with adaptive structures including wavenumber considerations," R. L. Clark and C. R. Fuller, "Active structural acoustic control with adaptive structures including wavenumber considerations," J. Intelligent Mater. Systems Structures 3(2), 296-315 (1992).

${ }^{4}$ F. Fahy, Sound and Structural Vibration (Academic, Orlando, FL, 1987), pp. 61-63.

${ }^{5} \mathrm{G}$. Maidanik, "Vibrational and radiative classification of modes of a baffled finite panel," J. Sound Vib. 34 (4), 447-455 (1974).

${ }^{6} \mathrm{C}$. R. Fuller and R. A. Burdisso, "A wave number domain approach to the active control of structure-borne sound," J. Sound Vib. 148(2), 355-360 (1991).

${ }^{7}$ L. Meirovitch, Analytical Methods in Vibrations (Macmillan, New York, 1967).

${ }^{8}$ R. L. Clark and C. R. Fuller, "Characterization of Multiple Piezoelectric Actuators for Structural Excitation," J. Acoust. Soc. Am. 90 (1), 346-357 (1991).

${ }^{9}$ C. E. Wallace, "Radiation Resistance of a Baffled Beam," J. Acoust. Soc. Am. 51(3), 936-945 (1972).

${ }^{10}$ S. J. Elliott, I. M. Stothers, and P. A. Nelson, "A multiple error LMS algorithm and its application to the active control of sound and vibration," IEEE Trans. Acoustic Speech Signal Process. ASSP-35, 14231434 (1987).

"B. Widrow and S. D. Stearns, Adaptive Signal Processing (Prentice-Hall, Englewood Cliffs, NJ, 1985).

${ }^{12} \mathrm{~J}$. S. Vipperman, R. A. Burdisso, and C. R. Fuller, "Active control of broadband structural vibration using the LMS adaptive algorithm," accepted for publication in the J. Sound Vib. (1992).

${ }^{13}$ Piezo Systems, Piezoelectric Motor/Actuator Kit Manual, Piezoelectric Products Inc., Cambridge, MA (1987). 